\title{
Evaluación Personalizada del Trabajo de Preparación para la Experimentación en Física mediante Técnicas de Monitorización.
}

José Daniel Sierra Murillo ${ }^{a}$

aUniversidad de La Rioja; Departamento de Química; Área de Física Aplicada; Complejo Científico-Tecnológico; C/ Madre de Dios 53; 26006-Logroño; daniel.sierra@unirioja.es.

\begin{abstract}
The main objective of this Teaching Innovation Project (TIP) is to properly evaluate the preparation work of each of the people who are part of an experimental group before it is put into practice in the Physics Laboratory. To do this, this work of prior preparation is intended to monitor through intelligent audiovisual resources (smartphone) that can be integrated into a Virtual Space 3.0 (VS-3.0). This monitoring could be done "live" (streaming) or by recording and storing the corresponding audiovisual file (mini-video) in a virtual reservoir accessible to all the actors involved: teachers and students. All with the invaluable help of the "Flipped Learning" Methodology, within the mentioned VS-3.0. This TIP highlights the importance of the use of Information and Communication Technology (ICT), not only in leisure ambits, but in other university training / learning, as well as the recognition of some of the philosophies of the Bologna 2020 Project, such as the continuous updating of the teaching-learning processes.
\end{abstract}

\section{Keywords:}

Improvement of the Personal Preparation Work Evaluation; Experimentation in Physics; Audiovisual Monitoring; Methodology "Flipped Learning"; Virtual Space 3.0.

\section{Resumen}

Este Proyecto de Innovación Docente (PID) tiene como principal objetivo poder evaluar adecuadamente el trabajo de preparación de cada una de las personas que forman parte de un grupo experimental antes de su puesta en práctica en el Laboratorio de Física. Para ello, dicho trabajo de preparación previa se pretende monitorizar a través de recursos audiovisuales inteligentes (smartphone) que puedan integrarse en un Espacio Virtual 3.0 (EV-3.0). Esta monitorización podría realizarse "en vivo" (streaming) o mediante la grabación y almacenamiento del correspondiente fichero audiovisual (mini-vídeo) en un reservorio virtual accesible a todos los actores implicados: profesores y alumnos. Todo ello con la inestimable ayuda de la Metodología "Flipped Learning", dentro del mencionado EV3.0. En este PID se pone de manifiesto la importancia del uso de las Tecnologías de la Información y la Comunicación (TIC), no sólo en ámbitos de ocio, sino en otros de formación/aprendizaje universitario, así como el 
reconocimiento de alguna de las filosofias del Proyecto Bolonia 2020, como es la actualización continuada de los procesos de docencia-aprendizaje.

\section{Palabras clave:}

Mejora de la Evaluación del Trabajo de Preparación Personal; Experimentación en Física; Monitorización Audiovisual; Metodología "Flipped Learning”; Espacio Virtual 3.0.

\section{Introducción}

A lo largo de mi carrera docente e investigadora, en diversos ámbitos teóricoexperimentales, llevo detectando defíciencias en la preparación teórica y experimental de algunas de las personas que forman parte de un pequeño grupo experimental, y que posteriormente deben llevar a cabo conjuntamente la correspondiente experimentación en un Laboratorio de Física Experimental (LFE).

Por esa razón, como ya se indica en el título del Proyecto de Innovación Docente (PID) que se expone en esta comunicación (Evaluación Personalizada del Trabajo de Preparación para la Experimentación en Física mediante Técnicas de Monitorización), el propósito con el que nace este PID es la mejora en la valoración del trabajo de preparación personal para la Experimentación en Física mediante el seguimiento o monitorización personalizado de la preparación previa para un buen trabajo en el LFE por parte de cada uno de los miembros del grupo de trabajo experimental: (1) preparación teórica, (2) montaje del experimento, (3) toma de medidas, (4) análisis de las mismas y (5) conclusiones. No obstante, aunque sería de gran interés realizar el mismo tipo de seguimiento y evaluación de todas las etapas del trabajo experimental, en principio, aquí se propone realizarlo sobre la primera etapa (1) mediante técnicas de monitorización que son de uso cotidiano en nuestra Sociedad de la Información y la Comunicación (SIC): el smart phone.

A lo largo de la Historia de la Humanidad, las Experiencias Científico-Tecnológicas han mostrado su beneficio a un mejor desarrollo de una sociedad moderna. Se venían realizando, ya desde el S. XVII, en diversos ámbitos como academias, sociedades científicas e, incluso, espacios de acceso a todo tipo de personas. Por supuesto, han sido de gran utilidad por su carácter docente y de aproximación a la senda de la experimentación. Instituciones como la universidad, institutos de investigación, etc. pueden encontrar en éstas una herramienta de gran interés docente e investigador. Sin embargo, aunque existen países de nuestro entorno que han visto en ellas su gran interés (González y Wagenaar, 2003; Wagenaar, 2018), en nuestro país el Proyecto Tuning de Física necesita un mayor acercamiento a la práctica docente generalizada, también con una perspectiva de iniciación a la experimentación científica y aplicación tecnológica. Aunque debe valorarse el trabajo realizado en algunos ámbitos universitarios españoles, entre los que cabe destacar el llevado a cabo en la Facultad de Ciencias Físicas de la Universitat de València (Ferrer, 2018). En 
este proyecto vinculado con la Física, hay involucrada una vasta comunidad universitaria entre profesores y alumnos, incluidos alumnos de Máster.

Aunque cada vez ocurre menos en nuestro país, sigue sucediendo que alumnos universitarios (algunos ni pueden matricularse o lo hacen compatibilizando trabajo y estudio) de grados relacionados con la ciencia y la tecnología llegan por vías en las que su formación en Física es mejorable. Sucede, sobre todo, en la etapa de Educación Secundaria Obligatoria (ESO), pero también en el Bachiller. A veces, por una inadecuada elección de asignaturas que preparen al alumno su capacitación para abordar los estudios universitarios antes mencionados. Aquí conviene recordar lo conveniente de aconsejar ciertas asignaturas indispensables, dentro de una cierta flexibilidad en la elección, aunque no siempre es posible en todos los Institutos de Enseñanza Secundaria (IES). En muchos casos, a esto se une la imposibilidad de plasmar de manera experimental lo poco o mucho aprendido desde una perspectiva teórica muy necesaria. El que un alumno pueda diseñar un experimento, más o menos sencillo, relacionado con la materia tratada de manera teórica, le abre puertas, no solo al mejor entendimiento de los fundamentos de la citada materia, sino de posibles mejoras y nuevos retos relacionados, en mayor o menor medida, con el primer experimento. No solo tiene que ver con la mejora del aprendizaje, sino con una nueva mirada hacia adelante, también con una mejora de "La Perspectiva Científica" (Russell, Bertrand, 1981). Desde un punto de vista global, la utilización de Experiencias Físicas (EF) permite elaborar todo un proceso cognitivo de observación racional, en el que es de gran interés que los alumnos emitan conclusiones en función de los sistemas físicos estudiados en el ámbito teórico, muy importante en la comprensión del Método Científico. Sobra decir que este método ofrece una gran mejora en la participación del alumnado de forma activa en su propio proceso de aprendizaje. Además de en su posible futuro profesional, en ámbitos de la investigación básica y/o aplicada $\mathrm{y}$, también, en el emprendimiento tecnológico, fundamental en la Sociedad del Siglo XXI.

Se conoce suficientemente que la calidad docente en la universidad, sean enseñanzas científicas, técnicas, etc., así como la mejora del proceso de aprendizaje por parte del alumnado, se puede obtener mediante métodos activos. (Alba, J., Torregrosa, C. y Del Rey, R., 2015) Queda claro en lo expuesto anteriormente que la propia naturaleza de la experimentación física posibilita y facilita la participación activa del alumnado, así como su participación individual y/o como miembro de un grupo de trabajo con el que colaborar en todo el proceso de aprendizaje. En este punto conviene recordar que se está trasladando una parte de la responsabilidad de su aprendizaje desde el profesor al alumno: Metodología "Flipped Learning" (MFL), (Prieto, 2017). Por experiencia del que suscribe, muy interesante cuando se complementa con el manejo de las TICs dentro de un Espacio Virtual 3.0 (EV-3.0). Las Webs 3.0 también se han venido a denominar Webs semánticas (W3C, 2013). (Figura 1) 


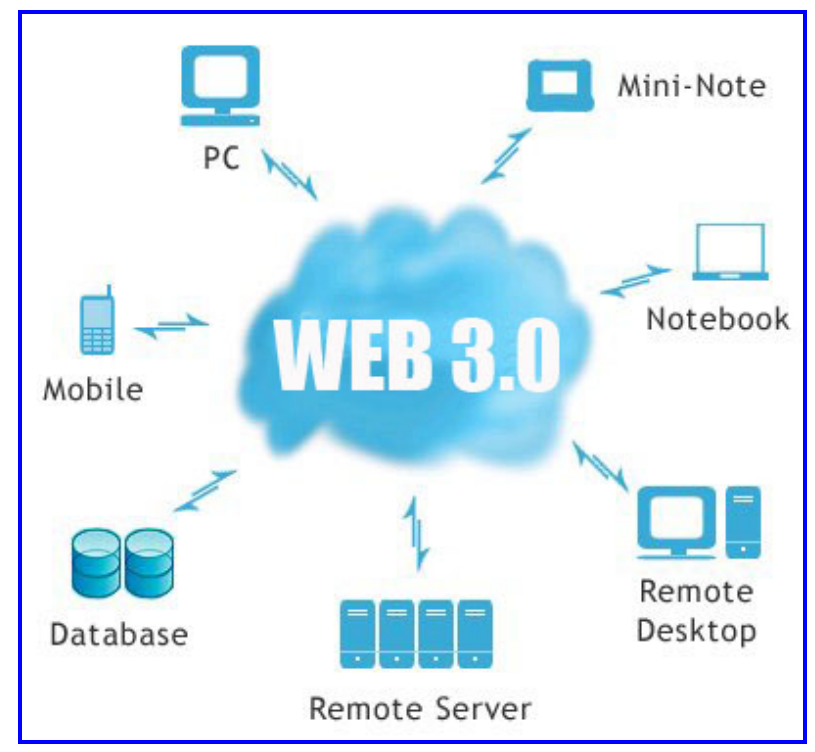

Figura 1. Visión esquemática de la Web 3.0. (Fuente: Google Web 3.0)

Este EV-3.0 se utiliza cada vez más en nuestra sociedad. Es como una autopista dentro de Internet que proporciona unas serie de herramientas de gran interés científico-tecnológico para el alumnado del que se habla en este proyecto. También para que instituciones, empresas, etc. puedan compartir información muy diversa. (Mora H., Azorín, J., Jimeno, A., Sánchez, J. L., Pujol, F., García, J., Serra, J. A., Morell, V., Rives, M. F., Saval, M., García, A. y Orts, S., 2016) Este concepto de Web semántica constituye un complemento de la $\mathrm{Web}$ tradicional. En él, la información se dispone de manera estructurada para permitir una ágil y eficiente consulta y acceso, tanto por humanos como por máquinas (Silva, J. M., Mahfujur, A. S. Md. y El Saddik, A., 2008; Nacer y Aissani, 2014).

En cuanto a la educación superior, ésta ha evolucionado hacia objetivos presentes y futuros dentro de un sistema formativo de una sociedad moderna y cambiante en muchos y diversos ámbitos (González Mariño, 2008; Ortega, 2018):

- Evolucionan los procesos de aprendizaje, desde los entornos presenciales tradicionales hacia otros más actuales y diversos.

- Existe una creciente demanda de mayor diversidad en las competencias específicas y transversales (Bolonia, 2009), así como de que se amplíe la oferta de formación continua sobre diferentes tipos de habilidades (informáticas, etc.) necesarias en los mencionados procesos de aprendizaje.

Los espacios de aprendizaje han evolucionado y las instituciones educativas también. Todo ello ha hecho que deban reajustar sus sistemas de intercambio de información y su comunicación con la comunidad educativa. Cambios que traen consigo nuevas estructuras organizativas, con características modulares, flexibilidad y mayor capacidad de intercambio de información sobre entornos reales y virtuales. Entre otras, flexibilidad en sus 
procedimientos y en su estructura administrativa, de acuerdo a las necesidades de una nueva sociedad. (Ortega, 2018) En la actualidad, estas instituciones tienen que responder a este desafío. Deben revisar sus referentes y promover experiencias innovadoras. Pueden apoyarse en las TIC, modernizar las estrategias docentes de profesores y alumnos, entre otras cosas, para poder buscar, acceder, gestionar y compartir cada vez más información más o menos afín a las diferentes materias objeto de estudio, aprendizaje y experimentación. Todo esto forma parte de los procesos de mejora e innovación docente, investigadora, así como de su aplicación tecnológica y empresarial. (Mora H., Signes, M. T., De Miguel, G. y Gilart, V., 2015)

La universidad y su profesorado disponen de una experiencia en el ámbito de la enseñanza virtual en la que ha sido necesaria la participación activa de toda la comunidad universitaria. Además del correspondiente compromiso institucional con la Innovación Docente (ID) (Ramírez, 2018) y su puesta en valor al mismo nivel que la Investigación Específica (IE) tradicional en diversos ámbitos llevada a cabo por los mismos actores principales: PDI y alumnado en sus diferentes etapas universitarias y/o empresariales. En la universidad actual se trabaja muy intensamente por y para dicha IE, algunas veces incluso en detrimento de la docencia y su innovación. Las razones parecen obvias: su ejercicio es más valorado en la mayoría de los ámbitos institucionales. Parecería más aconsejable una valoración más equilibrada de una y otra, más cuando dichos procesos de ID implican habitualmente una mejora de toda actividad universitaria. Tanto en la docente (enseñanzaaprendizaje) como en la futura investigación básica y/o aplicada, así como en las mejoras metodológicas, relativas a competencias y a diversas habilidades, de interés para las empresas en las que el mencionado alumnado desarrollará todo su potencial. También es importante recordar y valorar la formación fundamental en los primeros cursos de los diferentes grados. Ésta forma parte de una necesaria, amplia y sólida base para un afianzado crecimiento del aprendizaje/conocimiento de un alumnado del Siglo XXI. Formación fundamental con competencias específicas y transversales, así como habilidades, necesarias y relacionadas con el necesario afán de mejora profesional de los actores implicados: PDI, alumnado, empresariado, etc.

"Los estudiantes, no solo deben ser buenos conocedores de cada una de las materias, sino también deben desplegar otras cualidades como la creatividad, el espíritu crítico y la capacidad para el aprendizaje continuo que esta sociedad en pleno Siglo XXI les solicita": Declaración Mundial para la Educación Superior en el Siglo XXI (Granados, 2011) y Declaración de Bolonia 2020 (Bolonia, 2009).

\section{Objetivos}

Como ya se indicó, el fundamental objetivo de este proyecto es conseguir monitorizar y evaluar el trabajo de preparación realizado por cada grupo de trabajo experimental a través de un recurso audiovisual inteligente que pueda integrarse en el arriba mencionado EV-3.0. Esta monitorización podría realizarse "en vivo" o mediante su grabación y almacenamiento del correspondiente fichero audiovisual (mini-vídeo) en un reservorio virtual accesible a 
todos los actores implicados: profesores y alumnos. A través de este seguimiento audiovisual es más eficiente y adecuada la evaluación del trabajo de preparación realizado por cada miembro del grupo de trabajo experimental.

En el desarrollo del proyecto se generará diverso material para el seguimiento/monitorización del trabajo de preparación previo a la experimentación en el LFE: guías metodológicas, guiones adaptados a los diferentes experimentos físicos planteados, material audiovisual trasmitido/grabado por los miembros del grupo de trabajo experimental que permita al profesor realizar el seguimiento necesario para una mejor valoración del nivel de preparación del grupo de trabajo experimental.

Debido al carácter multidisciplinar del proyecto, también se pretende como objetivo cercano conseguir adherir un número creciente de profesores afines a estas metodologías. A este objetivo puede ayudar el hecho de que la Física es una materia necesaria en todo tipo de disciplinas científicas y tecnológicas.

En la lista de objetivos de este proyecto deben indicarse también la mejora de las competencias del trabajo individual y grupal del alumnado, así como de estos con el profesor (Mazur, 1997). Todo ello con la inestimable ayuda de la arriba mencionada MFL, y dentro del EV-3.0, metodología y espacio virtual muy utilizado por el autor del proyecto. Para el adecuado desarrollo del mismo, es muy interesante que el alumnado disponga/adquiera algunas habilidades informáticas complementarias a las de comunicación audiovisual ya expuestas, y que son de gran importancia en los entornos científicos, tecnológicos y empresariales modernos en pleno Siglo XXI. (Karpicke y Blunt, 2011; Deslauriers, L., Schelew, E. y Wieman C., 2011; Freeman, S., Eddy, S. L., McDonough, M., Smith M. K., Okoroafor N., Jordt, H. y Wenderoth, M. P., 2014)

Por último, inherente a los anteriores objetivos, se consiguen otros de forma colateral, aunque no menos importantes. Entre otros, la comprobación de la importancia del uso de las TICs, no sólo en ámbitos de ocio, sino en ambientes dedicados a la formación/aprendizaje universitario en un EV-3.0. Además de la puesta en práctica de una de las filosofías del proyecto Bolonia 2020, como son los cambios de los procesos de docencia-aprendizaje. 


\section{Desarrollo de la innovación}

\section{Metodología}

La metodología a seguir se basa fundamentalmente en el desarrollo de los objetivos arriba expresados. Para ello, la colaboración entre los miembros de los grupos de trabajo experimental en la preparación de cada EF es vital. Así como la planificación de la trasmisión/grabación audiovisual de aquellos intervalos temporales del proceso de preparación de la experiencia que sean suficientemente significativos como para poder entender con claridad la idoneidad del mencionado proceso de preparación de la EF. Esa información audiovisual se trasladará en vivo al profesor o se le puede hacer llegar en forma de fichero (mini-vídeo) a través del EV-3.0. Dada la versatilidad de entorno virtual, podría utilizarse un Aula Virtual de la universidad a la que pertenecen los profesores participantes en el proyecto para poder llevar a cabo el desarrollo de este proyecto innovador. (Figura 2) Esta zona de intercambio administrada por el profesor permitiría compartir con cada uno de los grupos de trabajo experimental el material necesario inicialmente, además del generado por cada uno de ellos en el proceso de preparación. Facilitaría también posibles mejoras de los procesos de preparación, ya que, al poder observar con detalle el proceso de preparación experimental por diferentes profesores sin tener que reunirse de manera presencial, se agilizaría enormemente las optimizaciones de la preparación de los experimentos físicos.

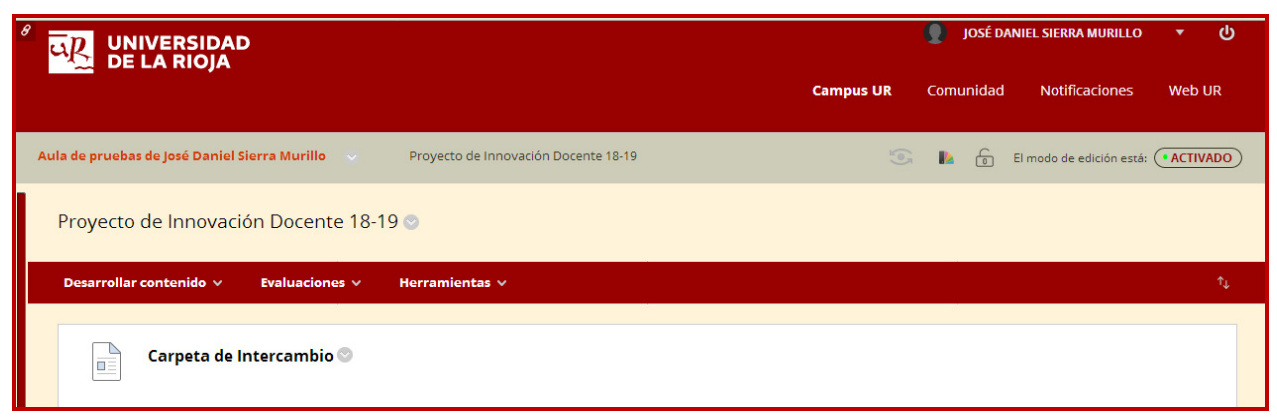

Fig. 2 Captura de pantalla de la zona de intercambio del Aula Virtual.

Se busca también la estandarización del proceso, que permita generalizar y extender el uso de diversas plataformas informáticas, con el objetivo de compatibilizar los trabajos autónomos y colaborativo del alumnado en el proceso de preparación de las EF mediante la arriba mencionada MFL sobre el EV-3.0, metodología y espacio virtual muy utilizado por el autor del proyecto con excelentes resultados. Todo lo anterior debe conllevar mayor facilidad de intercambio comunicativo entre los profesores implicados en este PID y el alumnado implicado en el mismo. Parte de ese entorno virtual podría estar formado por la Plataforma Virtual de la Universidad de La Rioja. Aunque también podría formar parte de 
dicho EV-3 .0 cualquier sistema de intercambio de información virtual actual o que pueda surgir en cualquier momento: WhatsApp (foros, grupos, etc.), diversas redes sociales, etc.

\section{Planificación y cronograma del proyecto}

La planificación del proyecto se realizará en tres fases. Se debe hacer notar el interés que se tiene en este PID, y otros anteriores del autor, en la mejora de la enseñanza tradicional hacia otro modelo en el que el protagonismo se reparta entre profesores y alumnado. Esta progresión lleva al alumnado hacia una mayor profundización en el aprendizaje de las materias objeto de investigación teórico-experimental, con la inestimable ayuda del EV-3.0 y la MFL en el óptimo desarrollo de los trabajos autónomo y colaborativo.

\section{Fase 1: Introducción metodológica.}

Se utiliza esta fase para comunicar al alumnado la metodología de trabajo en el desarrollo del PID. En los primeros días de la impartición de la asignatura de Física correspondiente (Mecánica, etc.), el profesor propondrá a cada grupo de trabajo experimental una tipología de experiencias para su estudio y preparación mediante la MFL. Cada grupo, a través del EV-3.0, podrá acceder a la información relativa a la materia a tratar de manera experimental: diversa documentación, referencias, etc.. Es sumamente interesante que cada grupo de trabajo experimental complemente la citada información con otra obtenida por ellos. Esto les puede ser de gran utilidad en el aprendizaje de las búsquedas bibliográficas contrastadas de forma autónoma (competencia trasversal), y también con el profesor.

\section{Fase 2: Trasmisión/grabación de la preparación de las Experiencias de Física.}

El proceso de preparación del trabajo experimental es una fase crítica y muy importante para el buen desarrollo de la experimentación en el LFE. Por ello, en este PID se pretende aquí que el trabajo autónomo y grupal (virtual y/o presencial) entre los miembros de cada grupo de alumnos se trasmita al profesor en vivo (streaming) o grabado en un fichero audiovisual (mini-vídeo) a través del mencionado EV-3.0 (Aulas Virtuales, WhatsApp, etc.). A lo largo de esta segunda fase, cada grupo de trabajo experimental puede consultar cualquier duda (específica de la materia, metodológica, etc.) con el profesor mediante tutorías presenciales y/o virtuales, y también con sus compañeros. El objetivo fundamental de esta fase es, a través de la monitorización "con pelos y señales" de todo el trabajo de preparación previa de la correspondiente EF prevista realizar posteriormente en el LFE, detectar defectos y fortalezas antes de llegar al laboratorio. De esta forma, debe aumentar la eficiencia y la calidad del trabajo presencial en el LFE.

Fase 3: Anexo audiovisual (mini-vídeo) a la memoria de cada Experiencia de Física. 
Como complemento a la memoria de cada Experiencia de Física, se anexaría el correspondiente fichero audiovisual (mini-vídeo, p.e., de unos 5 minutos) con una selección de los momentos más significativos del proceso de preparación del trabajo experimental que posteriormente se llevará a cabo en el LFE con mayor eficiencia y calidad.

$\checkmark$ Breve explicación de lo que va a consistir la Experiencia de Física.

$\checkmark$ Exposición "teatralizada" de las demostraciones teórico-prácticas requeridas en el guión de la EF correspondiente.

La distribución temporal del PID, o cronograma del proyecto, se distribuirá de tal forma que cada una de las EF pueda desarrollarse en periodos temporales aproximados de dos semanas, justo a la finalización de cada tema de Física relacionado con la misma.

\section{Resultados y Conclusiones}

Uno de los resultados obtenidos es la conclusión de la importancia de la utilización de las TIC en los procesos de aprendizaje universitario, mediante la MFL y en un EV-3.0. Además, que el perfil del PID enlaza con la filosofía de Bolonia 2020.

Por otra parte, se resuelve/concluye que se produce:

$\checkmark$ Una gran mejora del trabajo autónomo (responsabilidad personal) y colaborativo (corresponsabilidad) entre los alumnos y de alumnos-profesor a través del EV-3.0.

$\checkmark$ El progreso del alumnado en la utilización de:

- La conexión entre plataformas informáticas (hardware y software) con sistemas audiovisuales.

- Diversos sistemas audiovisuales utilizados para la transmitir y compartir la información relativa al proceso de preparación del posterior trabajo experimental en el LFE.

$\checkmark$ Una mejora cualitativa y cuantitativa del proceso de preparación del posterior trabajo experimental en el LFE por parte del alumnado, así como de sus resultados académicos. Incrementos del $10-20 \%$ en sus notas.

De gran interés es la generación de una base de información audiovisual sobre los diferentes procesos de preparación de diversos trabajos Teórico-Experimentales en Física.

\section{Referencias}

ALBA J., TORREGROSA C. y DEL REY R. (2015) Aprendizaje basado en proyectos: Primera experiencia en la asignatura de Física del Grado en Ingeniería de Telecomunicación, Sonido e Imagen. Universitat Politècnica de València Congreso IN-RED (2015).

BOLONIA. (2009). The Bologna Process 2020 - The European Higher Education Area in the new decade. Communiqué of the Conference of European Ministers Responsible for Higher Education.

DESLAURIERS, L., SCHELEW, E. y WIEMAN C.. (2011). Improved Learning in a LargeEnrollment Physics Class, Science, Vol. 332, pp. 862-864. DOI: 10.1126/science.1201783. 
Evaluación Personalizada del Trabajo de Preparación para la Experimentación en Física mediante Técnicas de Monitorización.

FERRER, C. (2018) Demostraciones experimentales de Física para el aula. URL: http://fisicademos.blogs.uv.es/?page id=29. Último acceso: 26/10/2018.

FREEMAN, S., EDDY, S. L., MCDONOUGH, M., SMITH M. K., OKOROAFOR N., JORDT, H. y WENDEROTH, M. P. (2014). Active learning increases student performance in science, engineering, and mathematics, Proc. Natl. Acad. Sci. USA, Vol. 111, pp. 8410-8415.

GONZÁLEZ, J. y WAGENAAR, R. (2003): Tuning Educational Structures in Europe. Informe Final - Proyecto Piloto, Fase 1, Bilbao, Universidad de Deusto.

GONZÁLEZ Mariño, J. C. (2008). TIC y la transformación de la práctica educativa en el contexto de las sociedades del conocimiento. Revista de Universidad y Sociedad del Conocimiento (RUSC). Vol. 5, n. ${ }^{\circ} 2$.

GRANADOS, J. (2011). The Challenges of Higher Education in the 21st Century, GUNi Newsletter, 5/11. (http://www.guninetwork.org/articles/challenges-higher-education-21st-century).

KARPICKE, J. D. y BLUNT, J. R. (2011). Retrieval Practice Produces More Learning than Elaborative Studying with Concept Mapping, Science, Vol. 331, pp. 772-775. DOI: 10.1126/science.1199327.

MAZUR, E. (1997). Peer Instruction: A User's Manual. New York: Prentice Hall Series in Educational Innovation.

MORA H., AZORÍN, J., JIMENO, A., SÁNCHEZ, J. L., PUJOL, F., GARCÍA, J., SERRA, J. A., MORELL, V., RIVES, M. F., SAVAL, M., GARCÍA, A. y ORTS, S. (2016). Nuevas tendencias Web 3.0 para la mejora de los procesos docencia-aprendizaje. Innovaciones metodológicas en docencia universitaria: resultados de investigación (pp.1543-1558). Alicante: Universidad de Alicante, Vicerrectorado de Estudios, Formación y Calidad, ICE.

MORA H., SIGNES, M. T., DE MIGUEL, G. y GILART, V. (2015). Management of social networks in the educational process, Computers in Human Behavior, Vol. 51, Part B, pp. 890-895. doi:10.1016/j.chb.2014.11.010.

NACER, H. y AISSANI, D. (2014). Semantic web services: Standards, applications, challenges and solutions, Journal of Network and Computer Applications, Vol. 44, pp. 134-151. DOI: 10.1016/j.jnca.2014.04.015.

ORTEGA, J. A. (2018). Planificación de ambientes de aprendizaje interactivos on-line: Las aulas virtuales como espacios para la organización y el desarrollo del teletrabajo educativo. URL: https://www.researchgate.net/profile/Jose Antonio15. Último acceso: 27/10/2018.

PRIETO MARTÍN, A. (2017-03-12). Decálogo de innovación metodológica para que los alumnos aprendan más y mejor en las asignaturas universitarias. Blog Profesor 3.0. URL: http://profesor3punto0.blogspot.com.es/2015/12/decalogo-de-innovacionmetodologica.html. Último acceso: 26/10/2018.

RAMÍREZ, M.S. (2018). Modelos y estrategias de enseñanzas para ambientes innovadores. Editorial Digital del Tecnológico de Monterrey.

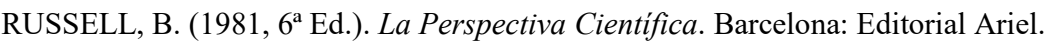

SILVA, J. M., RAHMAN, A. S. y EL SADDIK, A. (2008). Web 3.0: a vision for bridging the gap between real and virtual. Proceedings of the 1st ACM International Workshop on Communicability Design and Evaluation in Cultural and Ecological Multimedia Systems. (pp. 9-14). New York: ACM.

W3C. (2013). W3C Data Activity Building the Web of Data. URL: https:/www.w3.org/2013/data/. Último acceso: 26/10/2018.

WAGENAAR, R. (2018). Quality efforts at the discipline level: Bologna's Tuning process. En E. Hazelkorn, H. Coates and A.C. McCormick (Ed.), Research Handbook on Quality, Performance and Accountability in Higher Education, (pp. 275-289), Cheltenham, UK y Northampton, USA: Edward Elgar Publishing. 